\title{
Comparative analysis of the safety and efficacy of HBsAg-1018 versus HBsAg-Eng: a meta-analysis
}

\author{
LIN L. ZHANG, JING GUO, KAI DUAN
}

Wuhan Institute of Biological Products Co. Ltd., China

\begin{abstract}
Introduction: In addition to alum adjuvant, a wide diversity of adjuvants have been developed to enhance immune response of hepatitis $B$ virus $(H B V)$ vaccine in varying subjects, either in healthy vaccinators or subjects with hypo-immunity. In this context, a novel HBV vaccine HBsAg-1018, formulated with a toll-like receptor 9 agonist, was developed, and is currently in the phase of clinical trials. So, the first meta-analysis was performed to examine the safety and immune response of HBsAg-1018 among varying subjects.

Material and methods: On the basis of inclusion criterion, eligible studies that reported safety and immunogenicity induced by HBsAg-1018 vaccination in randomised, controlled trials (RCTs) were involved from three databases: PubMed, EMBASE, and the Cochrane Library, and further confirmed by two reviewers. Meta-analysis was conducted using RevMan 5.3. The pooled relative risk (RR) for safety and immunogenicity was calculated using random-effects or fixed-effects models according to the heterogeneity of included studies. The methodology quality of eligible studies was assessed using the Cochrane Handbook for Systematic Reviews of Interventions version 5.1.0.

Results: In total 5073 subjects administrated with HBV vaccine from four eligible publications were included in this meta-analysis. The data related to immunogenicity and safety post vaccination were pooled for meta-analysis. For safety, the combined RRs for adverse reactions were 0.98 (95\% CI: 0.89-1.08), 1.02 (95\% CI: 0.94-1.10) for AE, 0.88 (95\% CI: 0.70-1.10) for SAE, and 1.07 (0.12-9.17) for death. No statistical heterogeneity among RCTs was found $(p>0.05)$. For immunogenicity, at four weeks post vaccination, seroprotection rates (SPRs) in HBsAg-1018 were significantly superior to the conventional $H B V$ vaccine containing alum adjuvant, HBsAg-Eng (Engerix-B ${ }^{\text {, }}$, GlaxoSmithKline, Rixensart, Belgium) (RR: 4.35; 95\% CI: 3.35-5.65). Furthermore, superior immunogenicity of HBsAg-1018 was maintained with RRs up to 1.23 and $95 \%$ CI: 1.20-1.27 through 28 weeks post vaccination. However, there was considerable heterogeneity with $>80 \%$ I2 value $(p<0.05)$.

Conclusions: In comparison with HBsAg-Eng, HBsAg-1018 exhibited superior immune response and comparable safety profile with HBsAg-Eng in varying subjects. HBsAg-1018 is an effective and safe prophylactic measure to prevent $H B V$ infection.
\end{abstract}

Key words: hepatitis B, HBsAg-1018, HBsAg-Eng, meta-analysis.

(Centr Eur Immunol 2019; 44 (4): 455-462)

\section{Introduction}

Hepatitis B virus (HBV) is one of the most widespread pathogens throughout the world, which is thought to be 50 to 100 times more infectious than human immunodeficiency virus (HIV) [1][(CDC), 2009 \#1]. The global population infected with $\mathrm{HBV}$ has an estimated two billion population with over 240 million chronic carriers and 600,000 deaths each year [2][Organization, 2015 \#2]. HBV infection poses a serious public health concern, especially in highly endemic regions such as sub-Sahara Africa, East Asia, and central
Asian republics [3]. It is well known that vaccination is the most cost-effective measure to prevent infectious-disease spread. Since the first HBV vaccine was licensed in 1980s, mortality and morbidity in association with HBV infection have plunged dramatically [4-6]. A universal vaccination program against $\mathrm{HBV}$ in neonates and infants recommended by the World Health Organisation in 1991 has been implemented in Taiwan, Thailand, and China, and HBV vaccine efficacy in preventing mother-to-infant transmission, reducing chronic infections, and decreasing incidence of hepatocellular carcinoma [7-10] has been shown. 
Although the safety and protective effectiveness of current market-available HBV vaccines are documented thoroughly, a novel HBV vaccine with more immunogenic can further meet the requirements for hypo- and non-responders with inferior immunogenicity against the current HBV vaccine, such as older and obese individuals, dialysis patients, and immune-compromised hosts [11]. On the basis of published results, 1018 immuno-stimulatory sequence (1018 ISS), a toll-like receptor 9 agonist, has been demonstrated to enhance the immune response when being co-administered with antigens. Currently, 1018 ISS has been used as a novel adjuvant in an investigational HBV vaccine, HBsAg-1018. The results of all available clinical trials show that HBsAg-1018 would have the capability to elicit superior seroprotection in healthy vaccinators and hypo-responders to conventional HBV vaccines containing aluminium adjuvant [12]. In this context, we summarised the results from RCTs, and then we performed a systemic review of eligible studies to evaluate the immunogenicity and safety of HBsAg-1018 among a variety of subjects.

\section{Material and methods}

\section{Search and data extraction}

A comprehensive search strategy was used to retrieve eligible publications up to December 2017 across three databases: the Cochrane Library of Clinical Trials, EMBASE, and Pubmed. Additional relevant articles and reference lists of retrieved articles were searched by hand. The key terms used for retrieve were hepatitis B vaccine, HBV vaccine, ISS, 1018 ISS, HBsAg-1018, HBsAg-ISS, and HEPLISAVTM. Observational studies and clinical trials without HBsAg-Eng as a control group or enough methodology description in randomisation and blind method were excluded. English language articles were selected.

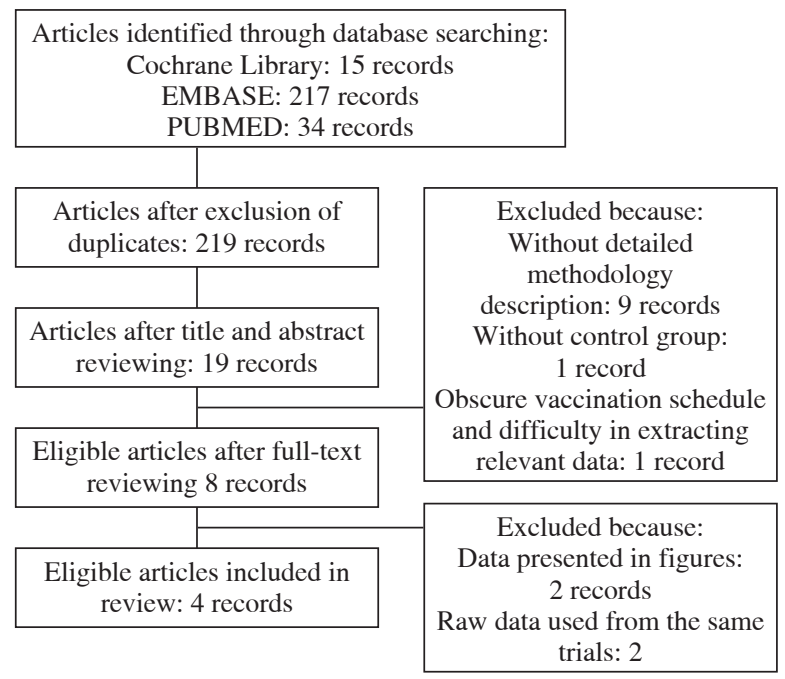

Fig. 1. Flow chat of articles evaluated for inclusion in the meta-analysis
All titles and abstracts were reviewed by two reviewers independently and duplicates were removed. Thereafter, we performed a full text review. Articles were included if they were recognised as eligible by both reviewers, and disagreements were judged by a third reviewer. From eligible publications, we extracted the data on study, subjects, intervention, control characteristics, and number and type of adverse events in both intervention and control groups. For safety, a percentage of subjects experiencing local and systematic reaction in one week after the first dose, adverse events (AE) from week 0 to week 28 , serious adverse events (SAE) from week 0 until week 52, and death, were extracted. For immunogenicity, the proportion of subjects achieving SPRs at 4, 28, and over 50 weeks were extracted separately.

\section{Methodology quality assessment}

The methodological quality of involved studies was assessed by two independent reviewers using standards recommended by the Cochrane Handbook for Systematic Reviews of Interventions version 5.1.0 [13]. According to the standards, studies were determined if they had low, unclear, or high risk of bias in random sequence generation, allocation concealment, blinding procedures, address of incomplete outcome data, and unselective reporting. No studies were excluded on the basis of bias.

\section{Data analysis}

The meta-analysis was performed with RevMan 5.3 (Cochrane Collaboration). For safety, RRs of the incident rate for adverse events were computed and pooled from each included study in two cohorts. The proportion of participants achieving anti-HBs $\geq 10 \mathrm{mIU} / \mathrm{ml}$ was considered to be seroprotective against HBV infection [14]. For immunogenicity, RRs of SPRs in two cohorts were calculated. The fixed effects or random effects models were chosen depending on heterogeneity between included studies (assessed by the I 2 statistic test). The I 2 values of $0-40 \%$, $30-60 \%, 50-90 \%$, and $75-90 \%$ indicate no important, moderate, substantial, and considerable heterogeneity, respectively. For significant heterogeneity of studies, the authors attempted to identify the sources of variation between studies by analysing the subgroup results and excluding them. Finally, the random effects model was used to pool the results without conducting sensitivity analysis for the limitation of a small amount of studies. In the two-tailed test, results were considered to be statistically significant with a $\mathrm{p}$ value of $<0.05$.

\section{Results}

\section{Study screening}

The flow chart of retrieving studies is shown in Figure 1. A total of 266 articles were identified from three 


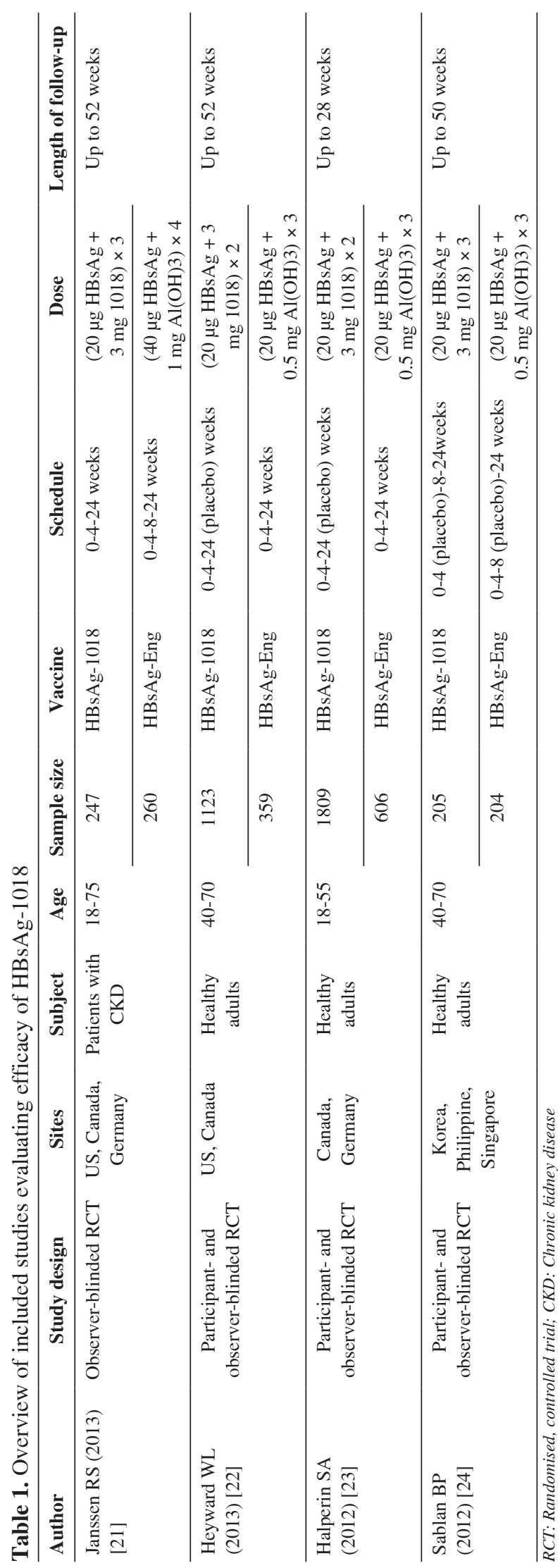

databases, with 47 articles excluded due to duplication. After referring to the titles and abstracts, 200 articles were considered ineligible. The remaining 19 articles were evaluated through full-text review. After reviewing full text, 13 publication were excluded, among which nine articles did not contain an adequate methodology description in randomisation and blind methods, one article was without HBsAg-Eng as a control group [15], one article had an obscure vaccination schedule and difficulty in extracting relevant data [16], and two articles had data that could not be obtained from figures or tables [17, 18]. For using raw data from same the RCTs, two articles were excluded $[19,20]$. Eventually, four RCTs were included in our meta-analysis [21-24].

\section{The profile of eligible studies}

Study characteristic are summarised in Table 1. All the four studies are RCTs [21-24]. In total, the studies included 5073 participants. RCTs were mainly undertaken in the USA, Canada, and Germany [21-23], except for one in Asian countries, Korea, Philippine, and Singapore [24]. Patients with chronic kidney disease (CKD) were enrolled in one RCT [21], and healthy subjects were enrolled in the others [22-25]. For HBsAg-Eng, subjects in two RCTs were vaccinated with $20 \mu \mathrm{g}$ HBsAg adjuvated with $0.5 \mathrm{mg}$ $\mathrm{Al}(\mathrm{OH}) 3$ per human dose at 0-4-24 weeks. In the other two RCTs, subjects were given four injections at 0-4-8-24 weeks. Furthermore, in one RCT by Janssen et al. [21], a human dose of $40 \mu \mathrm{g}$ HBsAg adjuvated with $1.0 \mathrm{mg}$ $\mathrm{Al}(\mathrm{OH}) 3$ per jab was adopted, and in another RCT by Sablan BP, four injections comprising three injections with $20 \mu \mathrm{g} \mathrm{HBsAg}$ adjuvated with $0.5 \mathrm{mg} \mathrm{Al}(\mathrm{OH}) 3$ per human dose at 0-4-24 weeks in addition to placebo vaccination at eight weeks were employed [24]. For HBsAg-1018, vaccine schedule of 0-4-24 weeks was used with $20 \mu \mathrm{g}$ HBsAg adjuvated with $3.0 \mathrm{mg} 1018$ per human dose in three RCTs [21-23] and placebo vaccination at 24 weeks in two RCTs [23, 24]. In an RCT by Sablan BP et al., the vaccination schedule comprised three injections with 20 $\mu \mathrm{g} \mathrm{HBsAg}$ adjuvated with $3.0 \mathrm{mg} 1018$ per human dose at 0,8 , and 24 weeks and one injection with placebo at four weeks [22-23]. All four RCTs [21-24] reported follow-up of immunogenicity at 4 and 28 weeks post vaccination with three RCT follow-ups over 50 weeks [21, 22, 24]. The wide age range of subjects from 18 to 75 years existed in four RCTs.

\section{Quality assessment}

The quality assessment of these studies was presented in Table 2. Only one RCT, referring to a computer-generated list, performed random sequence generation. In all RCTs, the concealment method was not described. Three trials were double blinded, and one RCT was the observer blinded. All RCTs were assessed to have low risks of in- 
Table 2. Quality assessment of included studies evaluating efficacy of HBsAg-1018

\begin{tabular}{lcccccc}
\hline Study & $\begin{array}{c}\text { Sequence } \\
\text { generation }\end{array}$ & $\begin{array}{c}\text { Allocation } \\
\text { concealment }\end{array}$ & Blinding & $\begin{array}{c}\text { Incomplete } \\
\text { outcome data }\end{array}$ & $\begin{array}{c}\text { Selective outcome } \\
\text { reporting }\end{array}$ & $\begin{array}{c}\text { Other source } \\
\text { of bias }\end{array}$ \\
\hline Janssen RS (2013) & Unclear risk & Unclear risk & Low risk & Low risk & Low risk & $/$ \\
\hline Heyward WL (2013) & Unclear risk & Unclear risk & Low risk & Low risk & Low risk & $/$ \\
\hline Halperin SA (2012) & Low risk & Unclear risk & Low risk & Low risk & Low risk & $/$ \\
\hline Sablan BP (2012) & Unclear risk & Unclear risk & Low risk & Unclear & Low risk & $/$ \\
\hline
\end{tabular}

A

HBsAg-1018 HBsAg-Eng Risk Ratio

Study or Subgroup Events Total Events Total Wight M-H, Fixed, 95\% CI

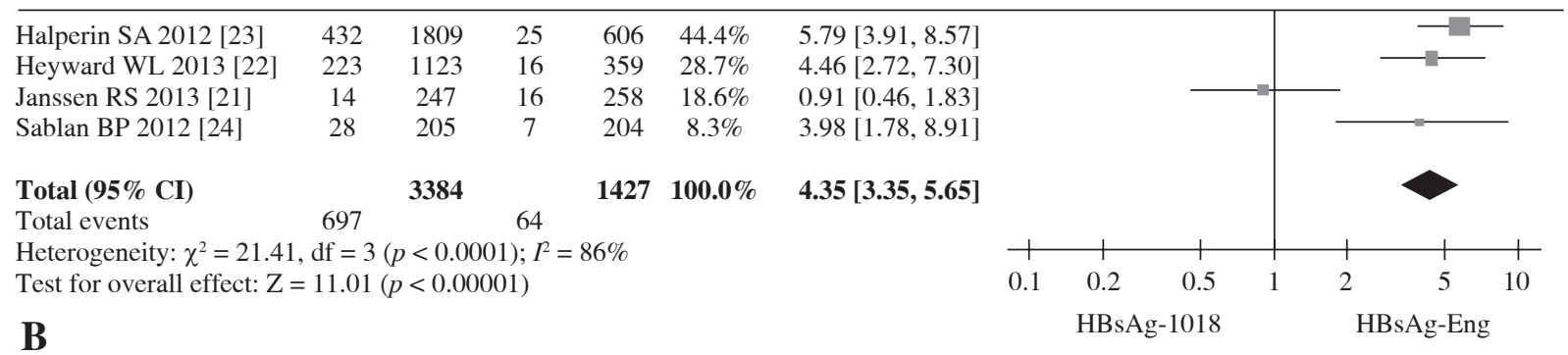

B

Risk Ratio

Study or Subgroup Events Total Events Total Wight M-H, Fixed, 95\% CI

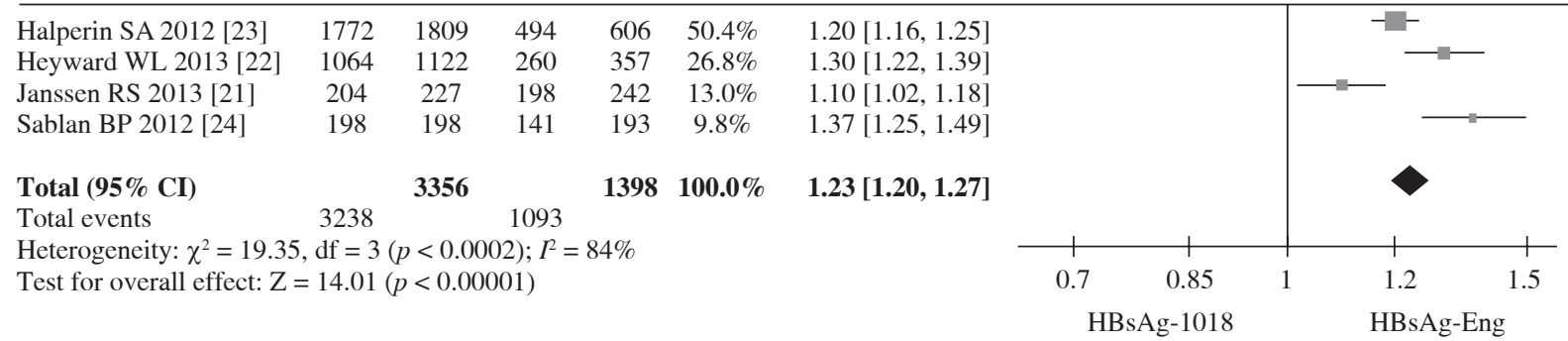

\section{C}

HBsAg-1018 HBsAg-Eng

Risk Ratio

Study or Subgroup Events Total Events Total Wight M-H, Random 95\% CI

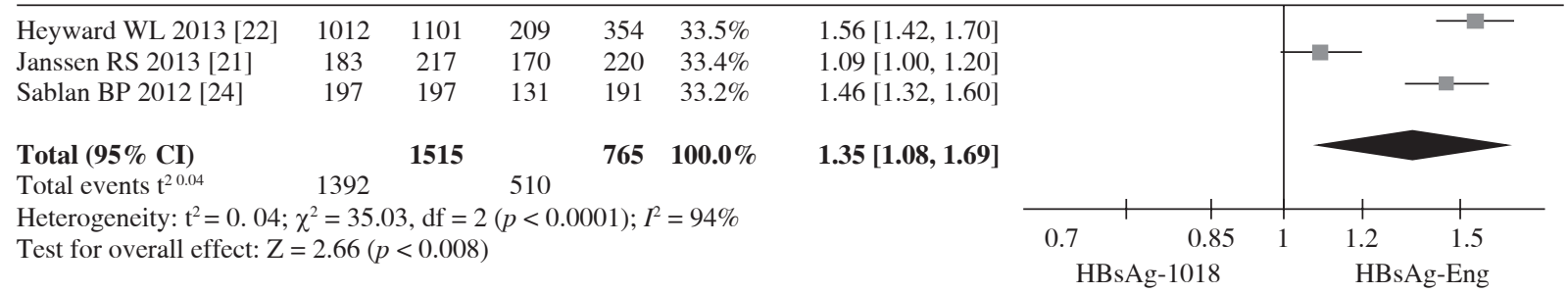

Fig. 2. Forest plot of sero-protection rates at 4 weeks (A), 28 weeks (B) and over 50 weeks (C)

complete outcome data and reporting bias. Overall, a moderate risk was observed in the methodological quality of all the RCTs.

\section{Immune response between HBsAg-1018 and HBsAg-Eng}

Despite SPRs being reported at varying time points in four included publications, we could extract successfully SPRs at 4 and 28 weeks from four publications and over
50 weeks from three publications post first jab in this meta-analysis. At four weeks post vaccination, pooled RRs of SPRs between HBsAg-1018 and HBsAg-Eng was 4.35 with 3.35-5.65 of $95 \% \mathrm{CI}$, indicating obviously favourable in the HBsAg-1018 group (Fig. 2A, raw data presented in Supplementary Table 1). Furthermore, the maintenance of superior immunogenicity in the HBsAg-1018 group could last for no less than half a year, during which RRs at 28 weeks was 1.23 (95\% CI: 1.20-1.27) (Fig. 2B, raw data presented in Supplementary Table 2). However, no dif- 


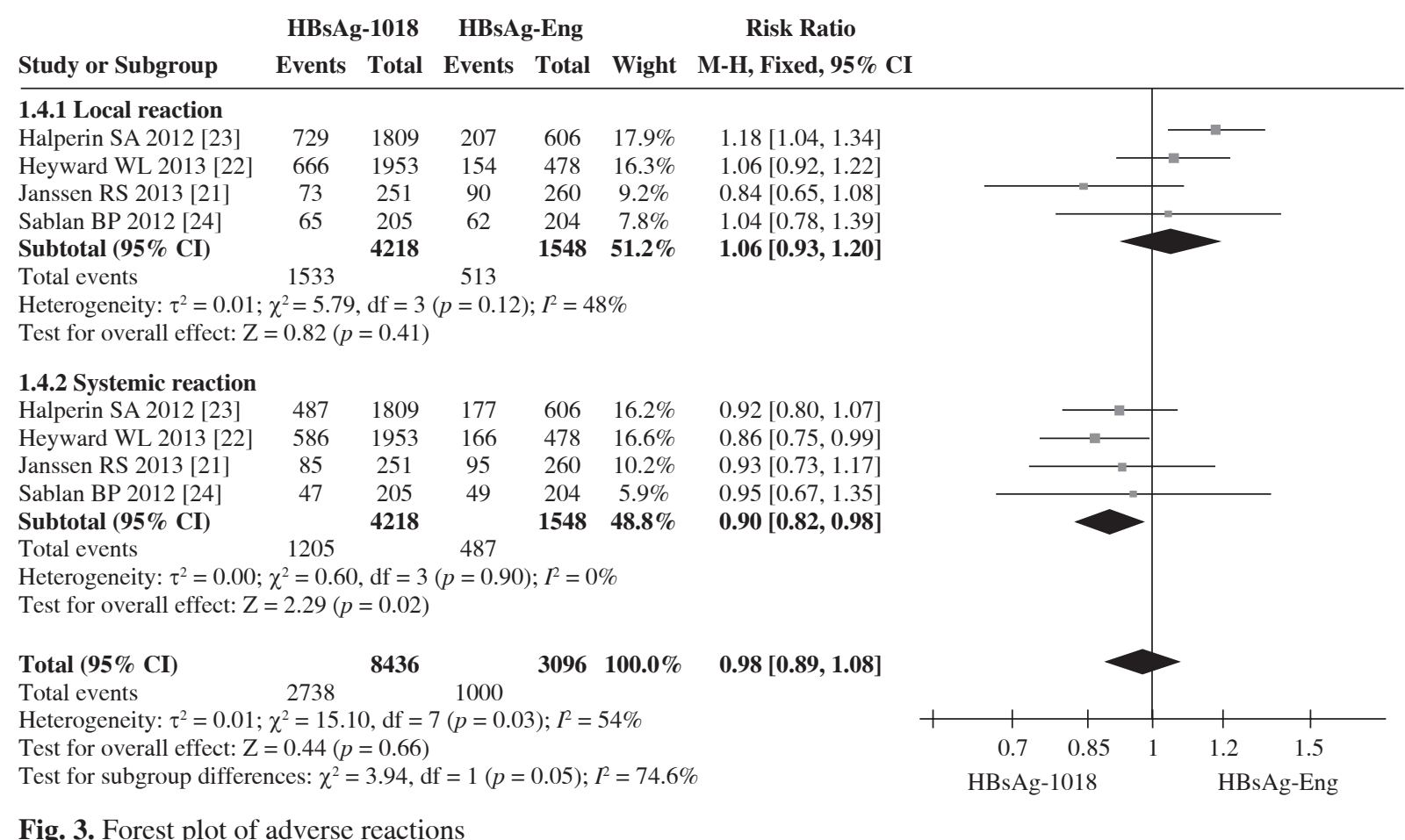

ference in immunogenicity was observed over 50 weeks, and RRs of SPRs over 50 weeks between HBsAg-1018 and HBsAg-Eng was 1.35 (95\% CI: 1.08-1.69) (Fig. 2C, raw data presented in Supplementary Table 3). In addition, there was obvious statistical heterogeneity of more than $80 \%$ I 2 at three different timepoints. An attempt to reduce heterogeneity or examining heterogenicity source was undertaken by deleting an RCT enrolling CKD patients as subjects. However, obviously statistical heterogeneity still existed (data not shown), documenting subject type might not be heterogenicity source in this meta-analysis.

\section{Safety}

In addition to adverse reactions, local reaction (injection pain, redness, and swelling), and systemic reactions (fever, headache, and fatigue), three RCTs reported detailed safety profiles about adverse events. Total RRs for adverse reactions post $\mathrm{HBV}$ vaccination crossed over one with $95 \%$ CI of $0.89-1.08$, indicating no statistical difference in the incidental rate of adverse reactions between HBsAg-1018 and HBsAg-Eng. Despite the presence of moderate heterogeneity $(\mathrm{I} 2=54 \%)$, there was a slight statistical significance ( $\mathrm{p}=0.03$ ) (Fig. 3, raw data presented in Supplementary Table 4). After pooling relevant data according to AE, SAE, and death individually, consistent results with those of adverse reactions were found (Figs. 4A, 4B, and 4C, raw data presented in Supplementary Tables 5,6 , and 7 , respectively). There was no difference in incidental rates of AE, SAE, and death, and RRs in inci- dental rates of AE, SAE, and death between HBsAg-1018 and HBsAg-Eng were 1.02 (95\% CI: 0.94-1.10, $p=0.14$ ), 0.88 (95\% CI: $0.70-1.10, p=0.83$ ), and 1.07 (95\% CI: $0.12-9.17, p=0.14)$, respectively. Moreover, no statistical heterogeneity was observed with $\mathrm{p}$ value more than 0.05 .

\section{Discussion}

Various attempts have been made to improve the protective efficacy of hepatitis B vaccine. These approaches included additional vaccine booster, intradermal vaccine route, and novel adjuvant systems [11]. In some studies, novel adjuvant systems, such as levamisole, granulocyte macrophage colony stimulating factor, interferon, and inmunoferon were proven to enhance the immunogenicity of HBV vaccine [25-28]. However, among patients with chronic diseases, the efficacy of adjuvant in HBV vaccine did not reach any agreement [29-34]. Four reviews on levamisole or GM-CSF showed that these adjuvants had the capability of improving the immune response against $\mathrm{HBV}$, especially in adults with end-stage renal disease [29-32]. And the one performed by Fabrizi exhibited that thymopentin, only injected at a higher dose, significantly enhanced the immunogenicity of HBV vaccine [33]. However, a subsequent analysis found that thymopentin would have a poor effectiveness in boosting immune response post HBV vaccination in CKD patients [34]. To date, it is the first meta-analysis to examine immunogenicity of HBsAg-1018 containing 1018 ISS. Furthermore, unlike other 
A

\begin{tabular}{|c|c|c|c|c|c|c|}
\hline & HBsAg & -1018 & HBsA & y-Eng & & Risk Ratio \\
\hline Study or Subgroup & Events & Total & Events & Total & Wight & M-H, Fixed, $95 \%$ \\
\hline Heyward WL 2013 [22] & 995 & 1968 & 255 & 481 & $33.1 \%$ & $0.95 \%[0.87,1.05]$ \\
\hline Janssen RS 2013 [21] & 195 & 254 & 198 & 262 & $32.5 \%$ & $1.02[0.92,1.12]$ \\
\hline Sablan BP 2012 [24] & 175 & 206 & 162 & 206 & $34.3 \%$ & $1.08[0.99,1.18]$ \\
\hline Total (95\% CI) & & 2428 & & 949 & $100.0 \%$ & $1.02[0.94,1.10]$ \\
\hline $\begin{array}{l}\text { Total events } \\
\text { Heterogeneity: } \tau^{2}=0.00 \\
\text { Test for overall effect: } Z\end{array}$ & $\begin{array}{c}1365 \\
\chi^{2}=3.98 \\
=0.41(p\end{array}$ & $\begin{array}{l}, \mathrm{df}=2 \\
=0.68)\end{array}$ & $\begin{array}{l}615 \\
=0.14\end{array}$ & $R_{2}$ & & \\
\hline
\end{tabular}

\section{B}

\begin{tabular}{|c|c|c|c|c|c|c|}
\hline \multirow[b]{2}{*}{ Study or Subgroup } & \multicolumn{2}{|c|}{ HBsAg-1018 } & \multicolumn{2}{|c|}{ HBsAg-Eng } & \multirow[b]{2}{*}{ Wight } & \multirow{2}{*}{$\begin{array}{c}\text { Risk Ratio } \\
\text { H, Fixed, 95\% C }\end{array}$} \\
\hline & Events & Total & Events & Total & & \\
\hline Heyward WL 2013 [22] & 76 & 1968 & 23 & 481 & $24.9 \%$ & $0.81[0.51,1.27]$ \\
\hline Janssen RS 2013 [21] & 68 & 254 & 76 & 262 & $67.0 \%$ & $0.92[0.70,1.22]$ \\
\hline Sablan BP 2012 [24] & 10 & 206 & 13 & 206 & $8.1 \%$ & $0.77[0.35,1.71]$ \\
\hline Total (95\% CI) & & 2428 & & 949 & $100.0 \%$ & $0.88[0.70,1.10]$ \\
\hline $\begin{array}{l}\text { Total events } \\
\text { Heterogeneity: } \tau^{2}=0.0 \\
\text { Test for overall effect: } 2\end{array}$ & $\begin{array}{l}154 \\
\chi^{2}=0.36 \\
=1.10(p\end{array}$ & $\begin{array}{l}\mathrm{df}=2 \\
=0.27)\end{array}$ & $\begin{array}{l}112 \\
p=0.8\end{array}$ & $R^{2}$ & & \\
\hline
\end{tabular}

C

HBsAg-1018 HBsAg-Eng

Study or Subgroup Events Total Events Total Wight M-H, Random 95\% CI

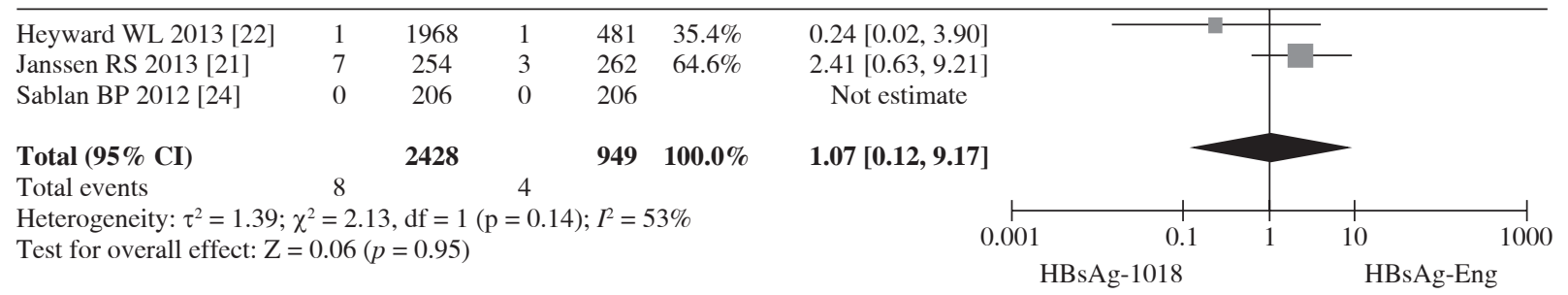

Fig. 4. Forest plot of AEs (A), SAEs (B) and death (C)

systematic reviews in which HBV vaccines without adjuvant were referred to as controls, in our meta-analysis the direct comparison between HBsAg-1018 and HBsAg-Eng was analysed.

Our results show that HBsAg-1018 is a safe and efficient vaccine. The results of the safety profiles showed that there were no comparisons in RRs of incidence rates of adverse reactions and adverse events between HBsAg-1018 and HBsAg-Eng. Furthermore, with regard to adverse reactions and adverse events, no statistical heterogeneity between HBsAg-1018 and HBsAg-Eng was observed in the included studies. Interestingly, when pooling RRs of SAE, I2 values fell to 0. All four RCTs adopted an observational period of seven days for local and systematic reactions post vaccination, and long-term safety profiles were assessed over 50 weeks by three RCTs. Obviously, our results proved that HBsAg-1018 exhibited comparable safety profiles in the short-term with that of HBsAg-Eng. However, for uncommon and serious adverse events, these four clinical trials enrolled a small-scale population, so the power of this meta-analysis to detect uncommon and serious adverse events was limited. In the future, large-scale clinical trials can be performed in order to monitor rare and severe adverse events post HBsAg-1018 vaccination.

In comparison with HBsAg-Eng, HBsAg-1018 elicited a superior antibody response in varying subjects. Moreover, the superiority persistently existed through 28 weeks. It is well known that immunogenicity and immune duration are crucial for protective efficacy post vaccination. For short-term immunogenicity, compared with HBsAg-Eng, HBsAg-1018 elicited superior immunogenicity at four weeks post vaccination with a higher proportion of subjects up to protective antibody concentration. However, the gap in the proportion of subjects with protective antibody concentration between HBsAg-1018 and HBsAg-Eng gradually narrowed over time. And no significant difference in immunogenicity between the two groups was observed over 50 weeks. By deletion of one RCT enrolling CKD patients, the heterogenicity level could not be reduced at different timepoints, indicating that subjects 
might not be a heterogenicity source. The varying vaccination schedule, subject age, and number of injections might be a heterogenicity source, but the small numbers of eligible studies limited the subgroup analysis. The above results documented that $\mathrm{HBsAg-1018}$ vaccination would stimulate robust and long-term immune response in a variety of subjects.

Our results should be interpreted with caution because of the following limitations. First, in spite of large-scale sample size of 5073 subjects, the number of eligible RCTs was small, and there might be a great source of bias. Second, a significant statistical heterogeneity of immunogenicity among these RCTs was observed, and the source of heterogeneity could not be identified by just deleting one RCT including CKD patients. Age and the number of injections were likely sources.

Finally, in the presence of varying types of data display, only SRPs at 4,28 , and over 50 weeks post vaccination could be extracted without protective antibody geometry mean titre. Thus, the comparison of antibody levels between HBsAg-Eng and HBsAg-1018 could not be evaluated in this meta-analysis.

\section{Conclusions}

In spite of the limitations discussed above, the results of our meta-analysis suggest that HBsAg-1018 has a similar safety profile to the the licensed HBV vaccine HBsAg-Eng and could provide robust seroprotection against the HBV infection among subjects in the short-term period.

The authors declare no conflict of interest.

\section{References}

1. CDC (2009): Hepatitis B FAQs for the Public.

2. WHO (2015): Guidelines for the Prevention, Care and Treatment of Persons with Chronic Hepatitis B Virus Infection.

3. Zanetti AR, Van Damme P, Shouval D (2008): The global impact of vaccination against hepatitis B: a historical overview. Vaccine 26: 6266-6273.

4. Chen DS (2009): Hepatitis B vaccination: the key towards elimination and eradication of hepatitis B. J Hepat 50: 805816.

5. Kim WR (2009): Epidemiology of hepatitis B in the United States. Hepatology 49 (Suppl): 528-534.

6. Chien YC, Jan CF, Kuo HS, et al. (2006): Nationwide hepatitis $B$ vaccination program in Taiwan: effectiveness in the 20 years after it was launched. Epidemiol Rev 28: 126-135.

7. Wait S, Chen DS (2012): Towards the eradication of hepatitis B in Taiwan. Kaohsiung J Med Sci 28: 1-9.

8. Liang X, Bi S, Yang W, et al. (2009): Epidemiological serosurvey of hepatitis B in China - declining HBV prevalence due to hepatitis B vaccination. Vaccine 27: 6550-6557.

9. Thursz M, Njie R, Lemoine M (2012): Hepatitis: global eradication of hepatitis B-feasible or fallacy? Nat Rev Gastroenterol Hepatol 9: 492-494.
10. Wichajarn K, Kosalaraksa P, Wiangnon S (2008): Incidence of hepatocellular carcinoma in children in Khon Kaen before and after national hepatitis B vaccine program. Asian Pac J Cancer Prev 9: 507-509.

11. Tajiri K, Shimizu Y (2015): Unsolved problems and future perspectives of hepatitis B virus vaccination. World J Gastroenterol 21: 7074-7083.

12. Eng NF, Bhardwaj N, Mulligan R, et al. (2013): The potential of 1018 ISS adjuvant in hepatitis B vaccines: HEPLISAV review. Hum Vaccin Immunother 9: 1661-1672.

13. Higgins JPT, Green S (2011): Cochrane Handbook for Systematic Reviews of Interventions Version 5.1.0. Available from: http://handbook.cochrane.org/.

14. Jack AD, Hall AJ, Maine N, et al. (1999): What level of hepatitis B antibody is protective? J Infect Dis 179: 489-492.

15. Halperin SA, Van Nest G, Smith B, et al. (2003): A phase I study of the safety and immunogenicity of recombinant hepatitis B surface antigen co-administered with an immunostimulatory phosphorothioate oligonucleotide adjuvant. Vaccine 21: 2461-2467.

16. Halperin SA, Ward BJ, Dionne M, et al. (2013): Immunogenicity of an investigational hepatitis B vaccine (hepatitis B surface antigen co-administered with an immunostimulatory phosphorothioate oligodeoxyribonucleotide) in nonresponders to licensed hepatitis B vaccine. Hum Vaccin Immunother 9: 1438-1444.

17. Halperin SA, McNeil S, Langley JM, et al. (2012): Safety and immunogenicity of different two-dose regimens of an investigational hepatitis $B$ vaccine (hepatitis B surface antigen co-administered with an immunostimulatory phosphorothioate oligodeoxyribonucleotide) in healthy young adults. Vaccine 30: 5445-5448.

18. Halperin SA, Dobson S, McNeil S, et al. (2006): Comparison of the safety and immunogenicity of hepatitis B virus surface antigen co-administered with an immunostimulatory phosphorothioate oligonucleotide and a licensed hepatitis B vaccine in healthy young adults. Vaccine 24: 20-26.

19. Janssen JM, Heyward WL, Martin JT, et al. (2015): Immunogenicity and safety of an investigational hepatitis B vaccine with a Toll-like receptor 9 agonist adjuvant (HBsAg-1018) compared with a licensed hepatitis B vaccine in patients with chronic kidney disease and type 2 diabetes mellitus. Vaccine 33: 833-837.

20. Janssen JM, Jackson S, Heyward WL, et al. (2015): Immunogenicity of an investigational hepatitis B vaccine with a toll-like receptor 9 agonist adjuvant (HBsAg-1018) compared with a licensed hepatitis B vaccine in subpopulations of healthy adults 18-70 years of age. Vaccine 33: 3614-3618.

21. Janssen RS, Mangoo-Karim R, Pergola PE, et al. (2013): Immunogenicity and safety of an investigational hepatitis $\mathrm{B}$ vaccine with a toll-like receptor 9 agonist adjuvant (HBsAg-1018) compared with a licensed hepatitis B vaccine in patients with chronic kidney disease. Vaccine 31: 5306-5313.

22. Heyward WL, Kyle M, Blumenau J, et al. (2013): Immunogenicity and safety of an investigational hepatitis B vaccine with a Toll-like receptor 9 agonist adjuvant (HBsAg-1018) compared to a licensed hepatitis B vaccine in healthy adults 40-70 years of age. Vaccine 31: 5300-5305.

23. Halperin SA, Ward B, Cooper C, et al. (2012): Comparison of safety and immunogenicity of two doses of investigational hepatitis B virus surface antigen co-administered with an immunostimulatory phosphorothioate oligodeoxyribonucleotide and three doses of a licensed hepatitis B vaccine in healthy adults 18-55 years of age. Vaccine 30: 2556-2563. 
24. Sablan BP, Kim DJ, Barzaga NG, et al. (2012): Demonstration of safety and enhanced seroprotection against hepatitis B with investigational HBsAg-1018 ISS vaccine compared to a licensed hepatitis B vaccine. Vaccine 30: 2689-2696.

25. Sali S, Alavian SM, Hajarizadeh B (2008): Effect of levamisole supplementation on hepatitis B virus vaccination response in hemodialysis patients. Nephrology 13: 376-379.

26. Lin C, Zhu J, Zheng Y, et al. (2010): Effect of GM-CSF in combination with hepatitis $B$ vaccine on revacination of healthy adult non-responders. J Infect 60: 264-270.

27. Jungers P, Devillier P, Salomon H, et al. (1994): Randomised placebo-controlled trial of recombinant interleukin- 2 in chronic uraemic patients who are non-responders to hepatitis B vaccine. Lancet 344: 856-857.

28. Perez-Garcia R, Perez-Garcia A, Verbeelen D, et al. (2002): AM3 (Inmunoferon) as an adjuvant to hepatitis B vaccination in hemodialysis patients. Kidney Int 61: 1845-1852.

29. Alavian SM, Tabatabaei SV (2010): Effects of oral levamisole as an adjuvant to hepatitis B vaccine in adults with end-stage renal disease: a meta-analysis of controlled clinical trials. Clin Ther 32: 1-10.

30. Fabrizi F, Dixit V, Messa P, et al. (2010): Meta-analysis: levamisole improves the immune response to hepatitis $B$ vaccine in dialysis patients. Aliment Pharmacol Ther 32: 756762.

31. Fabrizi F, Ganeshan SV, Dixit V, et al. (2006): Meta-analysis: the adjuvant role of granulocyte macrophage-colony stimulating factor on immunological response to hepatitis B virus vaccine in end-stage renal disease. Aliment Pharmacol Ther 24: 789-796.

32. Cruciani M, Mengoli C, Serpelloni G, et al. (2007): Granulocyte macrophage colony-stimulating factor as an adjuvant for hepatitis B vaccination: a meta-analysis. Vaccine 25: 709-718.

33. Fabrizi F, Dixit V, Martin P (2006): Meta-analysis: the adjuvant role of thymopentin on immunological response to hepatitis B virus vaccine in end-stage renal disease. Aliment Pharmacol Ther 23: 1559-1566.

34. Fabrizi F, Dixit V, Messa P, et al. (2012): Hepatitis B virus vaccine in chronic kidney disease: improved immunogenicity by adjuvants? A meta-analysis of randomized trials. Vaccine 30: 2295-2300. 